PROCEEDINGS OF THE

AMERICAN MATHEMATICAL SOCIETY

Volume 139, Number 7, July 2011, Pages 2435-2437

S 0002-9939(2010)10711-6

Article electronically published on December 23, 2010

\title{
MINIMAL NON-ELEMENTARY LIE ALGEBRAS
}

\author{
KRISTEN STAGG AND ERNEST STITZINGER
}

(Communicated by Gail R. Letzter)

\begin{abstract}
Minimal non-elementarty finite groups must be nilpotent. The Lie algebra analogue admits non-nilpotent examples. We classify them for complex solvable Lie algebras.
\end{abstract}

A group $G$ is called elementary if the Frattini subgroup of each subgroup of $G$ is the identity. Following this definition, a Lie algebra $L$ is elementary if the Frattini ideal of each subalgebra of $L$ is 0 . Elementary Lie algebras have been investigated by several authors; see the list of references. A group (Lie algebra) is minimal nonelementary if it is not elementary but each of the proper subgroups (subalgebras) is elementary. Kirtland has shown in [5 that a finite group is minimal non-elementary if and only if $G$ is either cyclic of order $p^{2}, p$ any prime, or a non-abelian $p$-group of order $p^{3}, p$ an odd prime. Hence all minimal non-elementary finite groups are $p$-groups. The analogous concept in Lie algebras admits non-nilpotent examples. In this note we find all such finite dimensional Lie algebras with nilpotent derived algebra over an algebraically closed field.

The Lie algebras considered here are solvable. Hence the Frattini subalgebra coincides with the Frattini ideal. Let $\phi(L)$ denote the Frattini subalgebra of $L$. For solvable Lie algebras over a field of characteristic 0 , the derived algebra is nilpotent; hence the theorem finds all minimal non-elementary Lie algebras in this case when the field is algebraically closed. For characteristic $p$, if $L$ is nilpotent of length 2, then the final term, $L_{w}$, in the lower central series is nilpotent, hence abelian. Then $L$ is the semi-direct sum of $L_{w}$ and a Cartan subalgebra $C$ of $L$ [1, Theorem 8]. Then $C$ is abelian and $L^{2}=L_{w}$. Hence the theorem applies to any Lie algebra of nilpotent length 2 over an algebraically closed field.

Lemma. Let L be a minimal non-elementary finite dimensional solvable Lie algebra with $L^{2}$ nilpotent.

(i) If $L$ is nilpotent, then $L$ is Heisenberg.

(ii) If $L$ is not nilpotent and $C$ is a Cartan subalgebra, then $L$ is the semi-direct sum $L=C+L^{2}$.

Proof. (i) Suppose that $L$ is nilpotent. Then $\phi(H)=H^{2}=0$ for every proper subalgebra $H$ of $L$ and $\phi(L)=L^{2}$. If $\operatorname{dim}\left(L / L^{2}\right)>2$, then every pair of

Received by the editors January 28, 2010 and, in revised form, July 16, 2010.

2010 Mathematics Subject Classification. Primary 17B30.

Key words and phrases. Minimal non-elementary Lie algebras, Frattini subalgebras.

(C)2010 American Mathematical Society Reverts to public domain 28 years from publication 
elements of $L$ are in a proper subalgebra of $L$ and hence commute. Thus $L$ is abelian, a contradiction. Hence two elements, $x$ and $y$, generate $L$. Since $\left\langle x, L^{2}\right\rangle$ and $\left\langle y, L^{2}\right\rangle$ are proper subalgebras of $L$, they are abelian and $L^{2} \subseteq Z(L)$. Hence $L=\langle x, y, z=[x, y]\rangle$ and $L$ is Heisenberg.

(ii) Suppose that $L$ is not nilpotent. Let $C$ be a Cartan subalgebra of $L$. As on page 57 of [4], $L$ decomposes as a vector space direct sum of $C$ and $C_{1}$ where $\left[C, C_{1}\right]=C_{1}$, the Fitting decomposition of $L$ with respect to $C$. Both $C$ and $L^{2}$ are nilpotent proper subalgebras of $L$; hence they are abelian. Since $C_{1} \subseteq L^{2}$, it follows that $C_{1}$ is an ideal of $L$. If $C_{1} \neq L^{2}$, then $C^{2} \cong\left(L / C_{1}\right)^{2} \cong$ $L^{2} / C_{1} \neq 0$, a contradiction. Hence $C_{1}=L^{2}$ and $L=C+L^{2}$ is a semi-direct sum.

Theorem. Let $L$ be a finite dimensional Lie algebra over an algebraically closed field $K$ and suppose that $L^{2}$ is nilpotent. Then $L$ is minimal non-elementary if and only if $L$ has a basis $x, y, z$ with multiplication $[x, y]=\alpha y+z,[x, z]=\alpha z$ and $[y, z]=0$, where $\alpha \in K$.

Proof. Assume $L$ is minimal non-elementary. If $L$ is nilpotent, then it has the desired presentation by part (i) of the Lemma. If $L$ is not nilpotent, then by part (ii) of the Lemma, $L$ is the semi-direct sum $L=C+L^{2}$, where $C$ is a Cartan subalgebra of $L$. Suppose that $\operatorname{dim} L / L^{2}>1$ and let $x \in C$. Then $H=\langle x\rangle+L^{2}$ is a proper subalgebra of $L$ and $\phi(H)=0$. In particular, $H$ is not nilpotent. If it were nilpotent, then $H$ would be abelian. Hence $x$ is in the center of $L$ and $\langle x\rangle$ is a direct summand of $L$, which is a contradiction. If $D$ is a Cartan subalgebra of $H$, then $H=D+H^{2}$ is a semi-direct sum using the same argument as in part (ii) of the Lemma. Since $L^{2}$ is abelian, the Fitting null component of $\operatorname{ad}(x)$ acting on $H$ is nilpotent; hence it is a Cartan subalgebra of $H$. Hence we can let $D$ be the Fitting null component. Since $\phi(H)=0$, Proposition 1 of [7] yields that $D$, hence $\operatorname{ad}(x)$, acts completely reducibly on $H$ since $x \in D, H=D+H^{2}$ and $D$ is abelian. Since $K$ is algebraically closed, $\operatorname{ad}(x)$ acts diagonally on $H$ and on the ideals $L^{2}$ and $\phi(L)$ that are contained in $H$. These results hold for all $x \in C$ and, since $C$ is abelian, the $\operatorname{ad}(x)$ are simultaneously diagonalizable on $L^{2}$ and on $\phi(L)$. Let $\left\{x_{1}, \ldots, x_{n}, y_{1}, \ldots, y_{t}\right\}$ be a basis of these common eigenvectors where the $x_{i} \in \phi(L)$ and $y_{i} \in L^{2}$, but $y_{i} \notin \phi(L)$. If $M=\left\langle x_{2}, \ldots, x_{n}, y_{1}, \ldots, y_{t}\right\rangle+C$, then $M$ is a maximal subalgebra of $L$. This is a contradiction since $x_{1} \notin M$, but $x_{1} \in \phi(L)$. Hence $\operatorname{dim} L / L^{2}=1$. Thus we can let $L=\langle x\rangle+L^{2}$.

We claim that $L^{2}$ is not the direct sum of two non-zero ideals of $L$. Suppose that $M$ and $N$ show this statement to be false. Let $A=\langle x\rangle+N$ and $B=\langle x\rangle+M$. Both $\phi(A)$ and $\phi(B)$ are 0 . Consider the set consisting of each maximal subalgebra of $A$ added to $M$. The intersection of the elements of this set is $M$, since $\phi(A)=0$ and $L$ is the semi-direct sum of $A$ and $M$. The Fitting null component $E \operatorname{of} \operatorname{ad}(x)$ acting on $A$ is nilpotent since $N$ is abelian. Hence $E$ is a Cartan subalgebra of $A$. Then $A=E+A^{2}$ is a semi-direct sum, $E$ is abelian and the action of $E$ on $A^{2}$ is the action of $\operatorname{ad}(x)$ on $A^{2}$. By Proposition 1 of [7], ad $(x)$ is diagonalizable on $A^{2}$ and also on $A$ since $E$ is abelian. Similarly, ad $(x)$ acts diagonally on $B$ and hence on $L^{2}$. By Proposition 1 of [7, $\phi(L)=0$ since $\langle x\rangle$ is a Cartan subalgebra of $L$. This contradiction establishes that $\operatorname{ad}(x)$ has only one Jordan block when acting on $L^{2}$. Hence there exists a basis $\left\{x_{1}, \ldots, x_{n}\right\}$ of $L^{2}$ such that $\left[x, x_{i}\right]=\alpha x_{i}+x_{i+1}$ for $i=1, \ldots, n-1$ and $\left[x, x_{n}\right]=\alpha x_{n}$, where $0 \neq \alpha \in K$. 
If $n>2$, then $B=\left\langle x, x_{2}, \ldots, x_{n}\right\rangle$ has $\phi(B)=0$ and ad $(x)$ acts diagonally on $B^{2}$ by Proposition 1 of [7, which contradicts the multiplication just given for $L$. Thus $n=2$ and the result holds. The converse is clear.

\section{REFERENCES}

[1] D. W. Barnes, On Cartan subalgebras of Lie algebras, Math. Z. 101 (1967), 350-355. MR0220785 (36:3837)

[2] C. Ciobanu, On a class of Lie p-algebras, Acta Math. Acad. Paedagog. Nyhazi (N.S.) 24 (2008), 279-285. MR2469949 (2009j:17018)

[3] C. Ciobanu, Some results on E-p-algebras, Proc. Rom. Acad. Ser. A Math. Phys. Tech. Sci. Inf. Sci. 10 (2009), 217-221. MR2539804

[4] N. Jacobson, Lie Algebras, Interscience Publishers, John Wiley \& Sons, New York, 1962. MR0143793 (26:1345)

[5] J. Kirtland, Finite groups with a minimal Frattini subgroup property, Glasgow Math. J. 45 (2003), 41-44. MR 1972691 (2004b:20033)

[6] M. Lincoln and D. Towers, The Frattini p-subalgebra of a solvable Lie p-algebra, Proc. Edinburgh Math. Soc. (2) 40 (1997), 31-40. MR1437809 (98a:17030)

[7] E. Stitzinger, Frattini subalgebras of a class of solvable Lie algebras, Pacific J. Math. 34 (1970), 177-182. MR0274540(43:303)

[8] E. Stitzinger, Theorems on Cartan subalgebras like some on Carter subgroups, Trans. Amer. Math. Soc. 159 (1971), 307-315. MR0280556 (43:6276)

[9] D. Towers, Elementary Lie algebras, J. London Math. Soc. (2) 7 (1973), 295-302. MR0376782 $(51: 12957)$

[10] D. Towers and V. Varea, Elementary Lie algebras and Lie A-algebras, Journal of Algebra 312 (2007), 891-901. MR2333190 (2008f:17008)

Department of Mathematics, North Carolina State University, Raleigh, North CaroLINA 27695

E-mail address: klstagg@ncsu.edu

Department of Mathematics, North Carolina State University, Raleigh, North CaroLINA 27695

E-mail address: stitz@math.ncsu.edu 